doi:10.13108/2020-12-2-21

\title{
ON MOMENT PROBLEM FOR ENTIRE FUNCTIONS GENERATED BY DOUBLY PERIODIC GROUP
}

\author{
F.N. GARIF'YANOV, E.V. STREZHNEVA
}

\begin{abstract}
We consider a lacunar problem for Stieltjes moments with an exponential weight. The solution is sought in the class of entire functions of exponential type, the indicator diagram of which is a some square. We construct nontrivial solutions of the corresponding homogeneous problem. This problem is reduced to the study of a linear total equation in the class of functions holomorphic outside four squares. At infinity, they have zero of a multiplicity at least three. Their boundary values satisfy the Hölder condition on any compact set containing no square vertices. At most logarithmic singularities are allowed at these vertices. The solution is sought in the form of an Cauchy type integral over the boundary of these squares with an unknown density. A method for regularizing the total equation is proposed. The condition of equivalence of this regularization is clarified. We find particular case when the obtained Fredholm equation of the second kind is solvable. In order to do this, we employ the principle of contracting mappings in a Banach space.
\end{abstract}

Keywords: regularization method, boundary value problems for elliptic functions, moments of entire functions of exponential type.

Mathematics Subject Classification: 30E05, 30E20, 30E25

\section{INTRODUCTION}

It is well-known [1, Ch. III, 3.1.2.5.] that

$$
\int_{0}^{\infty} x^{4 n+3} \exp (-x) \sin x d x=0, \quad n=0,1,2 \ldots
$$

The proposed elementary method of contour integration allows one to obtain more general identities

$$
L_{1}[F, n] \equiv \int_{0}^{\infty} x^{4 n+3} F(x) \exp (-x) \sin x d x=0, \quad n=0,1,2 \ldots
$$

Hereinafter, if else is not said, $F(z)$ is an entire function of the exponential type obeying the condition

$$
F(i z)=F(z)
$$

Its indicator satisfies the inequality

$$
h(\theta)<\cos (\theta)+\sin (\theta), \quad \theta \in[0, \pi / 2] .
$$

For constructing nontrivial solutions to a homogeneous problem

$$
\int_{0}^{\infty} x^{4 n+3} F(x) \exp (-x) d x=0, \quad n=0,1,2 \ldots
$$

F.N. Garif'yanov, E.V. Strezhneva, On moment Problem for entire functions generated by DOUBLY PERIODIC GROUP.

(C) Garif'Yanov F.N., Strezhneva E.V. 2020.

Submitted October 9, 2019. 
elementary methods are not applicable. One needs to reduce it to a linear difference equation with constant coefficients and here powerful classical methods for studying convolution operators are not applicable [2]. Problem (2) was completely considered in work [3]. The same concerns a corresponding inhomogeneous problem.

In work [4], there was studied the problem $L_{1}[F, n]=\beta_{n}, n=0,1,2 \ldots$, in the class of even entire functions of exponential type $F(z) \in A[5, \mathrm{Ch} . \mathrm{V}]$, whose indicator diagram was a segment $[-i, i]$ in the imaginary axis. In work [6], the problem

$$
\int_{0}^{\infty} x^{4 n+1} F(x) \exp (-x) \cos (x) d x=\beta_{n}, \quad n=0,1,2 \ldots
$$

was considered in the same class of entire functions of exponential type.

The aim of the present paper is to study the problems of momenta

$$
L[F, n] \equiv \int_{0}^{\infty} x^{4 n+1} F(x) \exp (-2 x) \sin (x) d x=\beta_{n}, \quad n=0,1,2 \ldots
$$

in the class of entire functions of exponential type $F(z)$ satisfying condition (1). We construct nontrivial solutions of the corresponding homogeneous problem, that is, as $\beta_{n}=0$ for all $n$. We stress that as in previous works $[3-4,6]$, we employ essentially the theory of elliptic functions.

The paper consists of three parts. In Section 2 we consider an auxiliary total equation in the class of the functions analytic outside four squares and having a zero at infinity of a multiplicity at least three. We propose a method of its regularization and find out a condition of its equivalence. In Section 3 we study particular cases of this equation, when it is possible to show an unconditional solvability of an obtained Fredholm equation of second kind. In Section 4, momenta problems (3) is reduced to this total equation. At that, we employ a Borel transform [7, Sect. 1, Subsect. 1].

\section{Regularization of total EQUATION}

Let $D_{1}$ be a square with vertices $t_{1}=\gamma(1+i), t_{2}=t_{1}+1, t_{3}=t_{2}+i, t_{4}=t_{1}+i$ and sides $l_{j}, j=\overline{1,4}$, counted in the order of passing a positively oriented boundary $\Gamma_{1}=\partial D_{1}, t \in l_{1}$, $\operatorname{Im} t=\gamma)$. Here $\gamma \in\left(2^{-1}, 1\right)$. We introduce four functions

$$
\sigma_{m}(z)=t_{m}+t_{m+1}-z ; \quad m=\overline{1,4} \quad t_{5}=t_{1} .
$$

They induce a Carleman shift $\alpha(t)=\left\{\sigma_{m}(t), t \in l_{m}\right\}$ mapping each side into itself with the change of the orientation. The centers of sides are fixed points of the shift.

We choose extra three squares $D_{j}=i^{j-1} D_{1}, j=\overline{2,4}$. The functions $\sigma_{m}(z)$ are defined by relation (4) only as $z \in D_{1}$. On the other squares we define them by the formula

$$
\sigma_{m}\left(i^{j-1} z\right)=i^{j-1} \sigma_{m}(z)\left(z \in D_{1}\right), \quad j=\overline{2,4} .
$$

Let $D=\bigcup_{k=1}^{4} D_{k}, \Gamma=\partial D, \Gamma_{j}=\partial D_{j}$. In this way, the shift $\alpha(t)$ is defined on the entire set $\Gamma$.

We are going to study a functional equation

$$
(V f)(z) \equiv \sum_{m=1}^{4} f\left[\sigma_{m}(z)\right]=g(z), \quad z \in D,
$$

under the following assumptions.

1) The solution $f(z)$ is holomorphic outside $D$ and at infinity it has a zero of multiplicity at least three. Moreover,

$$
f(i z)=-i f(z)
$$


Its boundary value $f^{-}(t)$ satisfies the Hölder condition on each compact set not containing the vertices of the squares. At the vertices, at most, logarithmic singularities are admitted. We denote such class of solutions by $B$.

2) The free term $g(z)$ is a piece-wise holomorphic function obeying (6), that is, $g(z)=g_{j}(z)$, $z \in D_{j}, j=\overline{1,4}$. Each function $g_{j}(z)$ is holomorphic in $D_{j}$ and its boundary condition satisfies $g_{j}^{+}(t) \in H_{\mu}\left(\partial D_{j}\right)$.

Let us clarify the formulation of the problem. To each square $D_{j}$, we associate the set $H_{j}=\bigcup_{m=1}^{4} \sigma_{m}\left(D_{j}\right)$. If $H_{j} \cap D_{k} \neq \emptyset$ as $k \neq j$, then problem becomes meaningless. We recall that a solution $f(z)$ is defined only outside $D$. Hence, $\gamma>2^{-1}$, which ensures that the squares are not too close one to another. On the other hand, we employ essentially that $H_{j} \cap H_{j+1} \neq \emptyset$, $j=\overline{1,4}, H_{5}=H_{1}$. This is why $\gamma<1$. We stress that problem (5) since the sets $\mathbb{C} \backslash H_{j}, j=\overline{1,4}$, are not connected.

We seek a solution to problem (5) as a Cauchy type integral:

$$
f(z)=\frac{1}{2 \pi i} \int_{\Gamma}(\tau-z)^{-1} \phi(\tau) d \tau, \quad z \notin \bar{D}
$$

with an unknown density obeying the conditions

$$
\phi(i t)=-i \phi(t)
$$

and

$$
\int_{\Gamma} \phi(\tau) d \tau=0 \Rightarrow \int_{\Gamma_{j}} \phi(\tau) d \tau=0 \quad \text { for all } j .
$$

Without loss of generality we assume that

$$
\phi(\tau)+\phi[\alpha(\tau)]=0
$$

Indeed, on each boundary $\Gamma_{j}$, the density $\phi(\tau)$ is defined up to a term $a_{j}^{+}(\tau)$, which is the boundary value of a function $a(z)$ holomorphic in $D_{j}$. By an appropriate choice of this function we can satisfy condition (10). This condition is regarded as the Carleman problem for the unknown function $a(z)$, which is solvable thanks to the locally-conformal gluing principle [8] and solvability condition (9).

We get

$$
\Leftrightarrow \quad(A \phi)(z)=\frac{1}{2 \pi i} \int_{\Gamma} \phi(\tau) E(z, \tau) d \tau=g(z), \quad z \in D
$$

where

$$
E(z, \tau)=\sum_{m=1}^{4}\left(\tau-\sigma_{m}(z)\right)^{-1} ; \quad z \in D
$$

Remark 1. Let us mention a relation of kernel (12) with the theory of elliptic functions. Let $\zeta(u)$ be a quasi-periodic Weierstrass function [9, Part 2, Ch. 1, Sect. 11] constructed by primitive periods $1 \pm i, u=\tau+z$. As $z \in D_{1}$, kernel (12) is the sum of four terms in the expansions of the zeta function into the series of primitive quotients closest to $D_{1}$.

In (11), we pass to the limit as $z \rightarrow t \in \Gamma$. In view of (10) we obtain an analogue of Sokhotskii-Plemelj formula:

$$
\left(A^{+} \phi\right)(t) \equiv 2^{-1} \phi(t)+(A \phi)(t)=g^{+}(t)
$$

where a singular integral $(A \phi)(t)$ is obtained by a formal change of the variable $z \in D$ by $t \in \Gamma$ in (11) and is treated in the Cauchy principal value. In relation (13) we replace the variable $t$ 
by $\alpha(t)$ and we deduct the obtained identity from the original one. In view of condition (10) we get:

$$
(T \phi) \equiv \phi(t)+\frac{1}{2 \pi i} \int_{\Gamma} K(t, \tau) \phi(\tau) d \tau=g^{+}(t)-g^{+}(\alpha(t)),
$$

and

$$
K(t, \tau)=E(t, \tau)+E[\alpha(t), \alpha(\tau)] .
$$

Lemma 1. Integral equation (14) is a Fredholm equation of second kind.

Proof. It is sufficient to confirm the boundedness of kernel (15) by a straightforward counting of all possible mutual location of the points $\tau$ and $t$ at the sides $\Gamma$.

Corollary 1. Integral equation (14) has finitely many solvability conditions.

Suppose that these conditions are satisfied. We make a back passage from integral equation (14) to original problem (5). In the same way how this was done in [10], one can show that there exists a solution $\phi(t)$ satisfying conditions (8) and (10). Then (14) implies that

$$
\left(A^{+} \phi\right)(t)-\left(A^{+} \phi\right)(\alpha(t))=g^{+}(t)-g^{+}(\alpha(t)),
$$

that is, $(A \phi)(z)=g(z)+c_{z}, z \in D$, since a doubly periodic function possessing no poles can be only constant. A piece-wise constant $c_{z}$ function is constant in each square and $c_{i z}=-i c_{z}$.

Theorem 1. Problem (5) has finitely many solvability conditions. These solvability conditions of integral equation (14) and one more condition:

$$
(A \phi)\left(z_{0}\right)=g_{1}\left(z_{0}\right)
$$

where $z_{0} \in D_{1}$ and this ensures the equivalence of the regularization.

Let all these assumptions to be satisfied and problem (5) be solvable. The set $H_{1} \cap H_{2}=H_{0}$ is a rectangle with vertices $\pm(\gamma-1)+\gamma i, \pm(\gamma-1)+(\gamma+1) i$, containing the segment $(\gamma i,(\gamma+1) i)$ in the imaginary axis. We choose a point $z \in H_{0}$. Then, employing the conditions of problem (5) on $D_{1}$, we have:

$$
f(z)=g_{1}\left(t_{1}+t_{4}-z\right)-f(z+1-i)-f(z+1+i)-f(z+2) .
$$

Exactly in the same way, employing the conditions of problem (5) on $D_{2}$, we obtain:

$$
f(z)=g_{2}\left(i t_{1}+i t_{2}-z\right)-f(z-1-i)-f(z-1+i)-f(z-2) .
$$

This implies

$$
\begin{gathered}
f(z+1-i)+f(z+1+i)+f(z+2)-f(z-1-i)-f(z-1+i) \\
-f(z-2)=g_{1}\left(t_{1}+t_{4}-z\right)-g_{2}\left(i t_{1}+i t_{2}-z\right) .
\end{gathered}
$$

Now let $\gamma<\operatorname{Im} z<2-\gamma$. Employing the conditions of problem (5) on squares $D_{3}$ and $D_{4}$, we get:

$$
\begin{aligned}
f(z+1-3 i) & +f(z+1-i)+f(z+2-2 i)-f(z-1-3 i)-f(z-1-i) \\
& -f(z-2-2 i)=g_{4}\left(2 i-i t_{1}-i t_{4}-z\right)-g_{3}\left(2 i-t_{1}-t_{3}-z\right) .
\end{aligned}
$$

As a result, we obtain:

$$
\begin{aligned}
f(z+1+i) & +f(z+2)-f(z-1+i)-f(z-2)-f(z+1-3 i) \\
& -f(z+2-2 i)+f(z-1-3 i)+f(z-2-2 i)=g_{0}(z),
\end{aligned}
$$

where

$$
g_{0}(z)=g_{1}\left(t_{1}+t_{4}-z\right)-g_{2}\left(i t_{1}+i t_{2}-z\right)-g_{4}\left(2 i-i t_{1}-i t_{2}-z\right)+g_{3}\left(2 i-t_{1}-t_{4}-z\right) .
$$

Relation (19) involves only the values of the function $f$ at the points located outside the square $D_{0}$ with vertices $(\gamma+1) i^{j}, j=\overline{1,4}$. 


\section{Study of integral Fredholm equation in SOME PARTiCUlar CASES}

In what follows we assume that $\gamma \geq 0.9$. Let us show that equation (14) is solvable. We consider the following homogeneous equation

$$
T \phi=0 .
$$

We assume that the operator $T$ is defined on the Banach space $\widetilde{C}(\Gamma)$. This is the set of the functions continuous on the closure of each side of the squares with a naturally induced norm:

$$
M=\max _{t \in \Gamma}|\phi(t)| .
$$

At that, for these functions, the vertices can be only the points of discontinuities of the first kind. Since $A(t, \tau)=A(\tau, t)$, we have $T^{\prime}=T$. A fundamental system of solutions of equation (21) can be chosen so that each involved function satisfies either condition (10) or an opposite condition $\phi(t)=\phi(\alpha(t))$, see [10]. Solutions with the latter condition are also orthogonal to the right hand side in (14) since (10) implies (9).

Lemma 2. The fundamental system of solutions of equation (21) contains no functions with property (10).

Proof. We assume the opposite and to be definite, we let $\gamma=0.9$. By property (10), instead of kernel (15), we take another kernel $K_{1}(t, \tau)=2^{-1}[K(t, \tau)-K(t, \alpha(\tau))]$ and we are going to estimate its absolute value from above. Thanks to the symmetry of $\Gamma$ it is sufficient to consider only two cases.

I. Condition (22) holds for $t \in l_{1}$. Then

$$
\begin{aligned}
A(t, \tau)= & (u-2.8-1.8 i)^{-1}+(u-3.8-2.8 i)^{-1}+(u-2.8-3.8 i)^{-1} \\
& +(u-1.8-2.8 i)^{-1} ; \quad u=\tau+T ; \quad \alpha(t)=2.8+1.8 i-t ; \\
& t=x+0.9 i ; \quad x \in[0.9,1.9] .
\end{aligned}
$$

1.1. $\tau \in l_{1}$; this implies that

$$
\begin{aligned}
K(t, \tau)= & (u-3.8-2.8 i)^{-1}+(u-2.8-3.8 i)^{-1}+(u-1.8-2.8 i)^{-1} \\
& -(u-1.8-0.8 i)^{-1}-(u-2.8+0.2 i)^{-1}-(u-3.8-0.8 i)^{-1},
\end{aligned}
$$

that is, $\left|K_{1}\right| \leqslant 0.13$.

1.2. $\tau \in l_{4}$, and in this case

$$
\begin{aligned}
K(t, \tau)= & (u-3.8-2.8 i)^{-1}+(u-2.8-3.8 i)^{-1} \\
& -(u-0.8-1.8 i)^{-1}-(u-1.8-0.8 i)^{-1},
\end{aligned}
$$

that is, $\left|K_{1}\right| \leqslant 0.08$.

1.3. $\tau \in l_{2}$. Thanks to the symmetry we have the same estimate $\left|K_{1}\right| \leqslant 0.08$.

1.4. $\tau \in l_{3}$. Then $K(t, \tau)=0$.

As $\tau \in \Gamma_{1}$, the kernel $E(t, \tau)$ contains the terms, the integral of which is treated in the sense of the Cauchy principal value. But kernel (15) is bounded and this is why, kernel $K$ is written explicitly above. As $\tau \notin \Gamma_{1}$, there are no such terms.

Let $\tau \in \Gamma_{2}$. We denote the lower, right, upper and left sides of the squares by $b_{j}, j=\overline{1,4}$, respectively. As $\tau \in l_{j}$, we have $\left|K_{1}\right| \leqslant c_{j}$, where $c_{1}=0.17 ; c_{2}=0.43 ; c_{3}=0.31 ; c_{4}=0.21$.

Let $\tau \in \Gamma_{3}$. In this case $c_{1}=0.04 ; c_{2}=0.06 ; c_{3}=0.07 ; c_{4}=0.08$.

Let $\tau \in \Gamma_{4}$. In this case $c_{1}=0.08 ; c_{2}=0.12 ; c_{3}=0.19 ; c_{4}=0.12$.

Since the sum of all these numbers is less than $2 \pi$, by the contracting mappings principle this implies that $\phi \equiv 0$ and this is a contradiction. 
II. Condition (22) is satisfied for $t \in l_{3}$. We observe that the point $t \in l_{3}$ is located further from the squares $D_{3}$ and $D_{4}$ than the point $t \in l_{1}$. This is why the above estimates for $t \in \Gamma_{3} \cup \Gamma_{4}$ become even better in comparison with case I and exactly in the same way we get $\phi \equiv 0$.

Finally, let $\gamma>0.9$. Then the ditances between the squares become greater than in the just considered case and this improves the estimates. The proof is complete.

Remark 2. It follows from the estimated established in the proof of Lemma 2 that it remains true also for some $\gamma<0.9$. The issue how small $\gamma$ can be is not considered in the present work.

Theorem 2. As $\gamma \geq 0.9$, problem (5) possesses the unique solvability condition (16).

Remark 3. For each function $g(z)$ we can find a constant $c$ such that for $\widetilde{g}(z)=g_{1}(z)+c$, $z \in D_{1}$, problem (5) is unconditionally solvable.

\section{Applications to momenta problem}

Here we discuss the application of problem (5) to momenta problem. Let an entire function $F(z)$ of exponential type be an upper function associated in the Borel sense with a lower function $f(z) \in B$. Generally speaking, its adjoint indicator diagram is the square $D_{0}$. It satisfies condition (1). We employ identity (6) and rewrite relation (19) as integral

$$
\int_{0}^{\infty} F(x) M(z, x) d x=g_{0}(z), \quad z \in D_{0}
$$

with the kernel

$$
\begin{aligned}
M(z, x)= & \exp (-x z-2 x)-i \exp (x i z+x i-x)+i \exp (x i z-x i-x) \\
& +\exp (z x-2 x)-i \exp (-x z i-x i-3 x)-\exp (2 i x-x z-2 x) \\
& +i \exp (x i-x i z-3 x)-\exp (x z-2 x-2 i x) .
\end{aligned}
$$

We stress that all exponentials are calculated at the points in the upper half-plane $\operatorname{Re} z>1+\gamma$. It is easy to confirm that for $n \neq 4 k+1, k=\overline{0, \infty}$ we have

$$
\left.\frac{\partial^{n}}{\partial z^{n}} M(z, x)\right|_{z=i}=g_{0}^{(n)}(i)=0
$$

and

where $z_{0}=2 \gamma(1+i)$. Let

$$
g_{0}^{(4 k+1)}(i)=-4 g_{1}^{(4 k+1)}\left(z_{0}\right)
$$

$$
g_{1}(z)=\beta_{0}-2 i \sum_{k=1}^{\infty} \frac{\beta_{k}}{k !}\left(z-z_{0}\right)^{k},
$$

and the convergence radius of this power series satisfies $R>\gamma \sqrt{2}$. We choose the coefficient $\beta_{0}$ so that problem (5) is solvable. Equating the like Taylor coefficients at the point $i$ in the left and right hand sides in identity (23), we find

$$
L[F, n]=\beta_{4 n+1} .
$$

Theorem 3. Momenta problems (25) in the class of entire functions $F(z)$ of exponential type associated in the Borel sense with the lower function $f(z) \in B$ is solvable under the condition

$$
\sqrt{2} \gamma e \varlimsup_{n \rightarrow \infty} \frac{\sqrt[4 n+1]{\left|\beta_{4 n+1}\right|}}{4 n+1}<1 .
$$

Let us construct a non-trivial solution to a homogeneous problem. It is sufficient to assume in formula (24) that $\beta_{4 n+1}=0$ and there exists a coefficient obeying $\beta_{n} \neq 0, n>0$. 
Remark 4. The case, when the adjoint indicator diagram is not the square $D_{0}$ but some smaller convex set $D_{0}^{\prime} \subset D_{0}$ is possible but is not interesting. In this case problem (5) is overdetermined. Condition (5) holds not only as $z \in D$, but also in the vicinity of the infinity. A necessary but not sufficient condition for this is the existence of an analytic continuation of $g_{1}(z)$ from $D_{1}$ into the vicinity of the infinity and having at the same time $g_{1}(\infty)=0$; for more details we refer to [11], [12].

\section{BIBLIOGRAPHY}

1. E.C. Titchmarsh. The theory of functions. Clarendon Press, Oxford (1932).

2. V.V. Napalkov. Convolution equations in multi-dimensional spaces. Nauka, Moscow (1982). (in Russian).

3. F.N. Garif'yanov. Stieltjes moments of entire functions of exponential type // Matem. Zamet. 67:5, 674-679 (2000). [Math. Notes. 67:5, 572-576 (2000).]

4. F.N. Garifyanov, E.V. Strezhneva. On a classic moment problem for entire functions // Lobachevskii J. Math. 39:6, 755-758 (2018).

5. B.Ya. Levin. Distribution of zeros of entire functions. Gostekhizdat, Moscow (1956). [Amer. Math. Soc., Providence, RI (1980).]

6. F.N. Garif'yanov, E.V. Strezhneva. On a system of entire functions of class A which is biorthogonal to a lacunar power system on the ray // Sibir. Matem. Zhurn. 58:1, 83-87 (2017). [Siberian Math. J. 58:1, 63-66 (2017).]

7. L. Bieberbach. Analytische Fortsetzung. Sringer, Berlin (1955). (in German).

8. Eh. I. Zverovich. A method of locally conformal gluing // Dokl. AN SSSR. 205:4, 767-770 (1972). [Sov. Math. Dokl. 13, 1003-1007 (1972).]

9. A. Hurwitz, R. Courant. Theory of functions. Nauka, Moscow (1967). (in Russian).

10. E.P. Aksent'eva, F.N. Garif'yanov. On the investigation of an integral equation with a Carleman kernel // Izv. VUZov. Matem. 4, 43-51 (1983). [Soviet Math. Iz. VUZ. 27:4, 53-63 (1983).]

11. F.N. Garif'yanov, S.A. Modina. On the four-element equation for the functions analytic beyond a trapezoid and its applications // Sibir. Matem. Zhurn. 52:2, 243-249 (2011). [Siberian Math. J. 52:2, 191-196 (2011)].

12. F.N. Garif'yanov, E.V. Strezhneva. Interpolation problems for entire functions induced by regular hexagons // Sibir. Matem. Zhurn. 59:1, 159-64 (2018). [Siberian Math. J. 59:1, 59-64 (2018)].

Farkhat Nurgayazovich Garif'yanov

Kazan State Power Engineering University

Krasnosel'skaya str. 51

420066, Kazan, Russia

E-mail: f.garifyanov@mail.ru

Elena Vasil'evna Strezhneva,

Kazan National Research Technical

University named after A. N. Tupolev - KAI

Karl Marx str. 10

420111, Kazan, Russia

E-mail: strezh@yandex.ru 